\title{
Upper GI Bleeding with Myocardial Infarction: Evaluation of Safety for Endoscopy
}

Roxanne G. Lim MD1; William J. Cobell MD1; Shoba Theivanayagam MD1; Todd W. Kilgore MD1; Michelle Matteson PhD1; Srinivas Puli MD²; Matthew L. Bechtold MD, FACG ${ }^{1}$

${ }^{1}$ Division of Gastroenterology - Department of Internal Medicine, University of Missouri , Columbia, MO; ${ }^{2} \mathrm{SSF}$ MG Gastroenterology, Peoria, Illinois

\section{BACKGROUND}

Upper gastrointestinal bleeding (UGIB) in the setting of acute myocardial infarction (MI) is a complex medical condition with substantial morbidity and mortality. The anemia due to the UGIB may exacerbate the MI or the anticoagulation for the $\mathrm{MI}$ may contribute to UGIB. In addition, both have many significant complications. Several studies have been performed in studying the safety of EGD after Ml; however, these studies vary in definitions and results. This study evaluated the safety and effect of EGD in patients with acute $\mathrm{MI}$ in a tertiary-care center.

\section{METHODS}

- Retrospective study (1/01 - 3/12).

- 86 patients who underwent EGD within 30 days of a MI at a single tertiary-care center.

- Patients identified by ICD-9 codes for MI (STEMI \& NSTEMI) and CPT codes for EGD.

- An extensive chart review was performed.

- Ml was defined as troponin I greater than laboratory reference "diagnostic of MI."

- Medical complications were defined as any documented changes in patients' symptoms, vital signs (BP $<90 / 60$ or $>180 / 100 \mathrm{mmHg}, \mathrm{HR}>100$ or $<60 \mathrm{bpm}, \mathrm{RR}>24 /$ minute, $\mathrm{O}_{2}$ saturation $<90 \%$, temperature $<35.0^{\circ} \mathrm{C}$ ), and telemetry (ventricular or atrial arrhythmias) within 24 hours of EGD.

- The study was statistically analyzed by the t-test and Fisher' $s$ exact test with significance indicated by $p$-value $<0.05$

\begin{tabular}{|c|c|c|cc|ccc|}
\hline & TOTAL & STEMI & NSTEMI & p-Value & Prior CC & No Prior CC & $p$-Value \\
Patients & 86 & 18 & 68 & & 28 & 58 \\
Peak Trop I (ng/mL) (mean) & $\mathbf{2 3 . 1 7} \pm \mathbf{5 9 . 1 7}$ & $40.71 \pm 40.04$ & $19.3 \pm 62.17$ & 0.10 & $23.63 \pm 31.82$ & $23.33 \pm 68.44$ & 0.49 \\
Time to Endoscopy (days) (mean) & $\mathbf{5 . 1 5} \pm \mathbf{6 . 0 0}$ & $2.01 \pm 2.27$ & $5.96 \pm 6.40$ & 0.006 & $5.06 \pm 5.49$ & $5.02 \pm 6.20$ & 0.49 \\
Apache II Score (n) (mean) & $\mathbf{1 2 . 7 3} \pm \mathbf{6 . 5 3}$ & $13.56 \pm 9.95$ & $12.51 \pm 5.33$ & 0.27 & $12.46 \pm 8.25$ & $12.86 \pm 5.57$ & 0.40 \\
Cardiac Cath/Total (\%) & $\mathbf{3 2 . 6 \%}$ & $77.8 \%$ & $20.6 \%$ & 0.08 & $100 \%$ & $0 \%$ & - \\
Complications (\%) & $\mathbf{3 1 . 4 \%}$ & $50.0 \%$ & $26.5 \%$ & 0.05 & $39.3 \%$ & $27.6 \%$ & 0.14 \\
\hline
\end{tabular}

Table 1: Summary of study results. $\mathrm{CC}=$ cardiac catheterization.

\section{RESULTS}

- Summary of results in Table 1.

- Complications were identified in 31.4\%. (Figure 1)

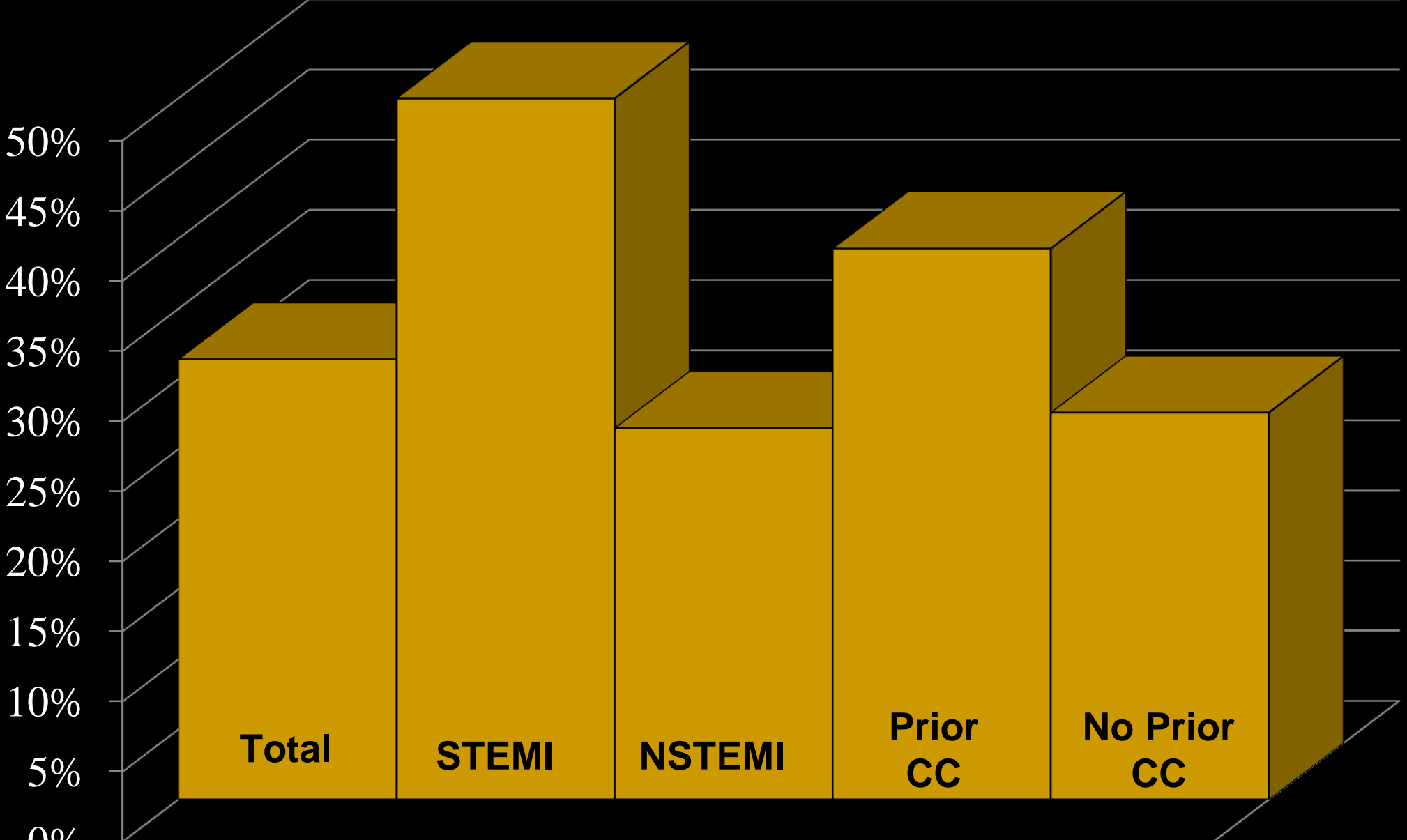

Figure 1: Comparisons of complications between type of $\mathrm{Ml}$ and cardiac catheterization (CC) status.

\section{- Hypotension (8) - Hypertension (2) \\ - Sinus Tachycardia (8) - Sinus Bradycardia (3) \\ - PVCs (1) - Hypothermia (1) \\ - Tachypnea (2) - Chest Pain (2)}

- STEMI patients underwent EGD sooner than NSTEMI patients $(p=0.006)$.

- No other significant differences were noted between types of Ml and cardiac catheterization status for peak troponin I, Apache II score, or complications.

\section{CONCLUSION}

EGD appears relatively safe for diagnosis and management of UGIB in patients with acute MI. Only minor complications were observed. The type of $\mathrm{Ml}$ and the need for cardiac catheterization do not result in a higher incidence of complications. 